\title{
Study of the Polarimetric Performance of a Si/CdTe Semiconductor Compton Camera for the Hitomi Satellite
}

\author{
Junichiro Katsuta $^{\mathrm{a}}$, Ikumi Edahiro ${ }^{\mathrm{a}}$, Shin Watanabe $^{\mathrm{b}}$, Hirokazu Odaka ${ }^{\mathrm{b}, \mathrm{c}}$, \\ Yusuke Uchida ${ }^{\mathrm{b}}$, Nagomi Uchida ${ }^{\mathrm{a}}$, Tsunefumi Mizuno, ${ }^{\mathrm{a}, *}$, Yasushi Fukazawa ${ }^{\mathrm{a}}$, \\ Katsuhiro Hayashi ${ }^{\mathrm{b}, \mathrm{e}}$, Sho Habata ${ }^{\mathrm{a}}$, Yuto Ichinohe ${ }^{\mathrm{d}}$, Takao Kitaguchi ${ }^{\mathrm{a}}$, \\ Masanori Ohno ${ }^{\mathrm{a}}$, Masayuki Ohta ${ }^{\mathrm{b}}$, Hiromitsu Takahashi ${ }^{\mathrm{a}}$, Tadayuki \\ Takahashi $^{\mathrm{b}}$, Shin'ichiro Takeda ${ }^{\mathrm{f}}$, Hiroyasu Tajima $^{\mathrm{e}}$, Takayuki Yuasa ${ }^{\mathrm{g}}$, \\ Masayoshi Itou ${ }^{\mathrm{h}}$, and SGD team \\ ${ }^{a}$ Hiroshima University, 1-3-1 Kagamiyama, Higashi-Hiroshima, Hiroshima 739-8526, Japan \\ ${ }^{b}$ Institute of Space and Astronautical Science, Japan Aerospace Exploration Agency, 3-1-1 \\ Yoshinodai Sagamihara, Kanagawa 252-5210, Japan \\ ${ }^{c}$ KIPAC, Stanford University, Stanford, CA 94305, USA \\ ${ }^{d}$ Tokyo Metropolitan University, 1-1 Minami-Osawa, Hachioji, Tokyo 192-0397, Japan \\ ${ }^{e}$ Nagoya University, Furo-cho, Chikusa-ku, Nagoya, Aichi 464-8601, Japan \\ ${ }^{f}$ OIST Okinawa Institute of Science and Technology Graduate University, 1919-1 Tancha, \\ Onna, Kunigami District, Okinawa 904-0495, Japan \\ ${ }^{g}$ RIKEN, 2-1 Hirosawa, Wako, Saitama 351-0198, Japan \\ $h$ Japan Synchrotron Radiation Research Institute (JASRI), SPring-8, 1-1-1 Kouto, \\ Sayo-cho, Sayo-gun, Hyogo 679-5198, Japan
}

\begin{abstract}
Gamma-ray polarization offers a unique probes into the geometry of the $\gamma$-ray emission process in celestial objects. The Soft Gamma-ray Detector (SGD) onboard the X-ray observatory Hitomi is a $\mathrm{Si} / \mathrm{CdTe}$ Compton camera and is expected to be an excellent polarimeter, as well as a highly sensitive spectrometer due to its good angular coverage and resolution for Compton scattering. A beam test of the final-prototype for the SGD Compton camera was conducted to demonstrate its polarimetric capability and to verify and calibrate the Monte Carlo simulation of the instrument. The modulation factor of the SGD prototype camera, evaluated for the inner and outer parts of the CdTe sensors as absorbers, was measured to be 0.649-0.701 (inner part) and 0.637-0.653 (outer part) at $122.2 \mathrm{keV}$ and $0.610-0.651$ (inner part) and 0.564-0.592 (outer part) at $194.5 \mathrm{keV}$ at varying polarization angles with respect to the detector. This indicates that the relative systematic uncertainty of the modulation factor is as small as $\sim 3 \%$.
\end{abstract}

Keywords: Polarimetric measurement, X-ray, $\gamma$ ray, Hitomi, SPring-8

\footnotetext{
* Corresponding author

Email address: mizuno@hep01.hepl.hiroshima-u.ac.jp (Tsunefumi Mizuno)
} 


\section{Introduction}

Gamma-ray polarization provides a unique probe into the geometry of the $\gamma$-ray emission process in celestial objects, such as the geometries of accretion disks around stellar-mass black holes and the magnetic field structures of pulsar and pulsar wind nebula. Detection of $\gamma$-ray polarization from cosmological sources also provides a stringent test of the vacuum birefringence effect resulting from some quantum gravity models. For example, INTEGRAL/IBIS measurements reported a change in the polarization angle after the Crab flare indicating magnetic field reconnection [1]. IKAROS/GAPs and INTEGRAL/IBIS observations of $\gamma$-ray polarizations from $\gamma$-ray bursts (GRBs) have also placed the most stringent limits by far on the vacuum birefringence effect to date [2 4] $]$. However, the INTEGRAL/IBIS measurements of the Crab polarization required observations of more than $10^{6} \mathrm{~s}$ because IBIS was designed as a coded mask instrument and was not optimized as a Compton camera. The GAPS measurements of the GRB polarization were marginal because of insufficient statistics due to the limited scattering angle coverage and the poor angular resolution under the severe resource constraints. To advance studies of $\gamma$-ray polarization from celestial objects, $\gamma$-ray instruments with higher polarization sensitivities are required.

The Soft Gamma-ray Detector (SGD) onboard the Hitomi satellite, the sixth Japanese X-ray observatory launched on February 17, 2016 [5, 6], is expected to provide 10 times better sensitivity in $60-600 \mathrm{keV}$ than past and current observatories [7-10]. The SGD achieves high sensitivities by suppressing the background using a combination of the BGO active shield and a Compton camera. Internal backgrounds that cannot be rejected by the active shield can be suppressed by requiring consistency between the incident direction of the $\gamma$ ray inferred by the Compton kinematics and the field of view defined by the collimator. The Compton camera consists of multiple layers of silicon ( $\mathrm{Si}$ ) sensors 11 13 surrounded by multiple layers of high-quality cadmium telluride (CdTe) sensors [14 16] to achieve good energy resolution and angular resolution for Compton kinematics. This design includes a compact camera with high detection efficiency enclosed in an active shield of a reasonable size that retains a sizable effective area.

The SGD also provides information on the polarization of the incident $\gamma$ rays because the Compton-scattering differential cross section depends on the azimuthal scattering angle with respect to the incident polarization vector. The SGD is also an excellent polarimeter because of its good angular resolution due to its highly segmented semiconductor sensors, and good scattering angle coverage [17, 18]

To conduct reliable polarimetric measurements in orbit, a precise characterization of the Compton camera for polarized $\gamma$ rays using a Monte Carlo (MC) simulation with a detailed model of the instrument is crucial. Therefore, we conducted a series of beam tests of prototypes for the SGD Compton camera 
using highly polarized $(\geq 99 \%) \gamma$ rays at the SPring- $\& 1$ synchrotron radiation facility to demonstrate the polarimetric capability of the instrument, and to verify and calibrate the MC simulator. As reported by [18], we evaluated the polarimetric performance of an early prototype for the SGD Compton camera, and verified that the observed azimuthal scattering angle distribution agrees well with the MC simulation. In November 2015, we conducted a beam test using the final-prototype for the SGD Compton camera whose design is the same as that of the flight hardware.

In this study, we demonstrate the polarimetric capability of the SGD Compton camera and verify and calibrate the MC simulation based on the experimental data from the 2015 beam test. In Section2, we briefly describe a method to derive polarization information using a Compton camera. A detailed description of the SGD Compton camera is given in Section 3. Section4 describes the setup and procedures for the beam test at SPring-8. In Section[5, we provide the experimental results and comparisons with the simulations.

${ }^{1}$ http://www.spring8.or.jp 


\section{Method of Polarimetric Measurement}

In this experiment, we adopted the standard method of polarimetric measurement for Compton polarimeters as described in Takeda et al. 18]. They derived the polarization information of the incident $\gamma$ rays from a measured azimuth scattering angle $(\phi)$ distribution based on the dependence of the Comptonscattering differential cross section on the azimuthal scattering angle $\eta$ with respect to the electric vector of the incident $\gamma$ ray:

$$
\frac{d \sigma}{d \Omega}=\frac{r_{e}^{2}}{2}\left(\frac{E^{\prime}}{E_{0}}\right)^{2}\left(\frac{E^{\prime}}{E_{0}}+\frac{E_{0}}{E^{\prime}}-2 \sin ^{2} \theta \cos ^{2} \eta\right),
$$

in which,

$$
E^{\prime}=\frac{E_{0}}{1+(1-\cos \theta) E_{0} / m_{e} c^{2}},
$$

where $r_{e}$ is the classical electron radius, $m_{e} c^{2}$ is the electron rest mass, $\theta$ is a scattering polar angle, and $E_{0}$ and $E^{\prime}$ are the energies of the incident and the scattered $\gamma$ rays respectively. Below we briefly describe the analysis procedure (see Takeda et al. [18] and references therein for details).

First, we measure the azimuthal angle distribution, $N_{\text {obs }}(\phi)$, using the $\mathrm{Si} / \mathrm{CdTe}$ Compton camera. Because the obtained distribution is affected by the detector response, $N_{\text {obs }}(\phi)$ needs to be divided by the azimuthal angle distribution for nonpolarized $\gamma$ rays, $N_{\text {iso }}(\phi)$ :

$$
N_{\text {cor }}(\phi)=\frac{N_{\text {obs }}(\phi)}{N_{\text {iso }}(\phi) /\left(\overline{N_{\text {iso }}}\right)},
$$

where $\overline{N_{\text {iso }}}$ is the average per angle bin.

By referring to Equation (1), we can see that the corrected azimuthal angle distribution follows a sinusoidal curve:

$$
N_{\text {cor }}(\phi)=A\left(1+Q \cos 2\left(\phi-\phi_{0}-\pi / 2\right)\right),
$$

where $A$ is the normalization, $Q$ is the so-called modulation factor, and $\phi_{0}$ is the direction of the polarization vector with respect to the electric vector of the incident photon [19]. By fitting the experimental data with this model formula, we can derive the polarimetric parameters, i.e., $\phi_{0}$ gives the polarization vector of the incident photon and $Q$ is related to the polarization degree $\Pi$ such that

$$
\Pi=\frac{Q}{Q_{100}},
$$

where $Q_{100}$ (i.e., the analyzing power) is the modulation factor for $100 \%$ linearly polarized $\gamma$ rays. The accuracy of the analyzing power affects the sensitivity of the polarimetric measurement of the detector. In Section 5, we evaluate the accuracy of $Q_{100}$ in the SGD Compton camera prototype via a comparison between the experimental data and the simulated data. 


\section{Compton Camera of SGD}

\subsection{Design}

Here we briefly summarize the design of the Compton camera (see [16] for details). The camera consists of top 32 layers of Si pixel sensors, bottom 8 layers, and 2 layers on each side of the CdTe pixel sensors, as shown in Figure 1 The bottom and the side layers are composed of $2 \times 2$ and $2 \times 3$ CdTe sensors respectively. The $\mathrm{Si}$ and the $\mathrm{CdTe}$ sensors have $16 \times 16$ pixels and $8 \times 8$ pixels, respectively, with respective thicknesses of 0.6 and $0.75 \mathrm{~mm}$. The pixel size is $3.2 \times 3.2 \mathrm{~mm}^{2}$ for both the Si and CdTe sensors. Figure 2 displays the geometry of the camera with definitions of instrumental (camera) $X-, Y$-, and $Z$-axes.

Each set of $8 \times 8$ pixels is read by a single ASIC (VATA450.3) mounted on a Front-End Card (FEC). The front-end electronics of the Compton camera consists of four groups of 52 FECs, one ASIC Driver Board (ADB), and one ASIC Control Board (ACB). Eight or six ASICs are daisy chained for readout and control. The ASICs are controlled by an FPGA on the ACB. These components are packed into a $12 \times 12 \times 12 \mathrm{~cm}^{3}$ aluminum box. One of the daisy chained readouts did not work for the camera used in this experiment; therefore, one fourth of the pixels in the eight Si layers cannot detect $\gamma$ rays. However, this is not the case for the flight-model SGDs.

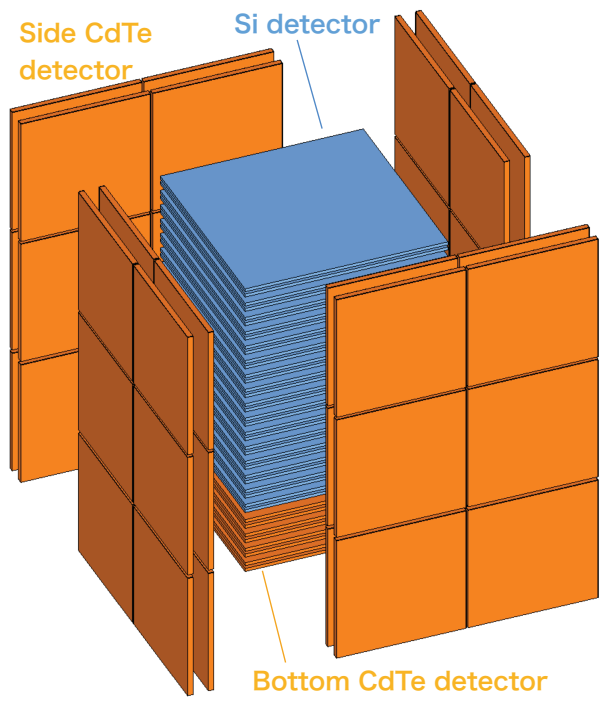

Figure 1: Conceptual image of the Compton camera. Positions of the Si and CdTe detectors are shown. 

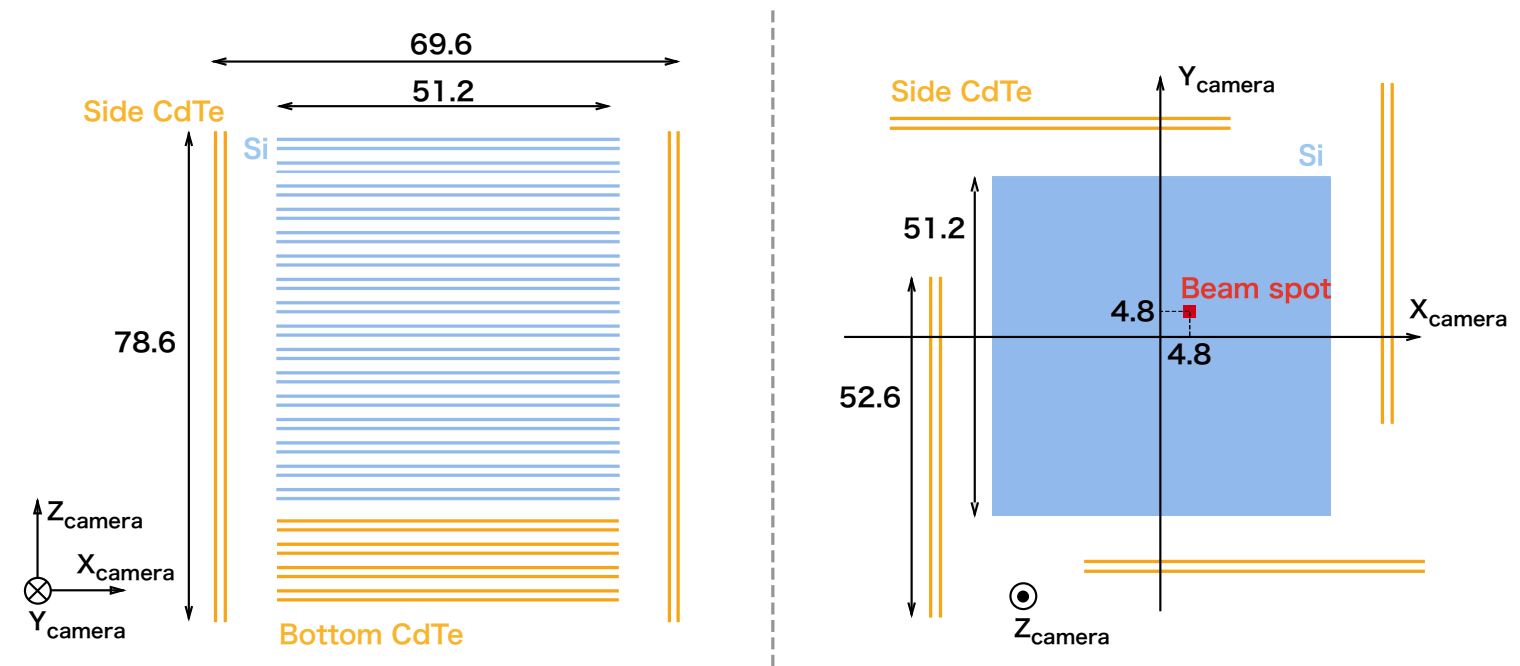

Figure 2: (Left) Side view of the Compton camera. The numbers are in millimeters. (Right) Overhead view of the Compton camera. The red square represents the incident beam spot (see Section 4 for details). 


\subsection{Event Reconstruction}

When observing weak objects in orbit, we require that each SGD event involve Compton scattering in the detector to apply Compton kinematics. The simplest case is that the event interacts twice in the camera: once via Compton scattering in a $\mathrm{Si}$ sensor and once via photo-absorption in a CdTe sensor. The time width to identify coincidence hits is $\sim 10 \mu s$, and typical low energy thresholds for hits in a Si sensor and a CdTe sensor are $\sim 5$ and $\sim 10 \mathrm{keV}$, respectively [16]. Once the locations and energies of the two interactions are measured, the Compton kinematics allows us to calculate the direction of the incident photon using the formula,

$$
\cos \theta=1+\frac{m_{e} c^{2}}{E_{1}+E^{\prime}}-\frac{m_{e} c^{2}}{E^{\prime}},
$$

where $E_{1}$ and $E^{\prime}$ are the energy deposited in the Compton site and the photoabsorption site, respectively, and are related to the incident photon energy such that $E_{0}=E_{1}+E^{\prime}$ [see also Equation (1)]. The deposit energies and positions are precisely measured with energy resolutions $(\Delta E)$ in full width at half maximum (FWHM) of $\sim 2$ and $\sim 3 \mathrm{keV}$ at $122 \mathrm{keV}$ for a Si sensor and a CdTe sensor, respectively, and a position resolution of $3.2 \mathrm{~mm}$. By requiring that the incident photon direction inferred from the Compton kinematics is consistent with its presence within the field-of-view of the SGD, we can distinguish target signals from the background. In addition, we apply a constraint on the topology and energetics of the detected events to efficiently reject background signals while retaining a large effective area. The details of the SGD background rejection can be found in [20].

\footnotetext{
${ }^{2}$ CdTe sensor has a low-energy tail in its spectrum and the energy resolution was evaluated with the tail taken into account in experiments. In simulation we modeled the spectrum with a gaussian for simplicity (see also Section 3.3).
} 


\subsection{Monte Carlo Simulator}

Unlike the monoenergetic pencil beam available at a synchrotron facility such as SPring- 8 , celestial $\gamma$ rays have a continuous energy spectrum and are irradiated on the entire detector surface area in orbit. Therefore, Monte Carlo simulations are essential to construct the detector response. We calculated the SGD instrumental response using ComptonSoft3 21], a Monte Carlo simulator that considers the detailed geometry of the Compton camera, including the passive materials. ComptonSoft includes Monte Carlo simulations and data analyses of Compton cameras, which are optimized for multilayer semiconductor Compton cameras in particular. It uses the Geant4 toolkit library [22, 23] as the Monte Carlo engine, and we adopted a low-energy electromagnetic physics option valid for polarized photons (class G4EmLivermorePolarizedPhysics is used as the physics list constructor in Geant4). We carefully built a mass model of the SGD Compton camera because a mass model is essential to produce the instrumental response accurately. We included not only the active detectors but also the passive materials, such as the supporting structures and the electrical circuit substrates in the mass model. Still there may be additional unaccounted for materials, e.g., small amounts of heavy metals inside the readout electronics, that affect photon absorption. We discuss this issue further in Section[5.

We performed simulations with $(2-4) \times 10^{7}$ photons to obtain statistically sufficient references for each measured energy (122.2 and $194.5 \mathrm{keV}$; see Section 4). The properties of the incident photons - energy, direction, and the size of the beam - were set to those in the experiment. The simulation code is capable of treating pixelated readout; therefore, we eliminated channels with malfunctions and/or with noises that could decrease the sensitivity to the polarization. The spectral response of each readout channel was found to be uniform; therefore, we assigned common energy-resolution parameters to all the readout channels of the same type of detector. The energy resolutions (FWHM) of the $\mathrm{Si}$ and the CdTe detectors are

$$
\begin{aligned}
& \Delta E_{\mathrm{Si}}=2.35 \times \sqrt{(0.80 \mathrm{keV})^{2}+(0.005 E)^{2}}, \\
& \Delta E_{\mathrm{CdTe}}=2.35 \times \sqrt{(0.82 \mathrm{keV})^{2}+(0.0079 E)^{2}},
\end{aligned}
$$

where $E$ is the energy deposited in a pixel. Even though it is known that a $\mathrm{CdTe}$ detector has a low-energy tail in its spectrum due to inefficient charge transport (see also, e.g., 21]), we can neglect this effect when examining the polarimetric response for this experiment because we applied a simple energy cut in the event selection, as described in Section 5 . We therefore simulated the spectrum of both Si and CdTe sensors assuming a gaussian response.

\footnotetext{
${ }^{3}$ https://github.com/odakahirokazu/ComptonSoft/
} 


\section{Setup and Procedure for the Experiment}

The experiment was conducted at the High Energy Inelastic Scattering Beamline BL08W 24 26] of SPring-8 from November 12-14, 2015. The experimental setup is illustrated in Figure 3 with definitions of $X$-, $Y$-, and $Z$-axes of the experiment. As shown in the figure, the camera was located in a thermostat bath, which kept the temperature at $-20^{\circ} \mathrm{C}$, the typical operational temperature of the SGD onboard the Hitomi satellite in orbit. For the rotation and alignment of the camera, we used motorized stages, as summarized in Table 1 .

The incident beam was lineally polarized at $\geq 99 \%$ in the horizontal direction and was irradiated perpendicular to the Si layers of the camera. Although the SGD is expected to provide good sensitivity in $60-600 \mathrm{keV}$ [7-10], we conducted the experiment using beam energy of 122.2 and then $194.5 \mathrm{keV}$. The beam energy of $122.2 \mathrm{keV}$ was selected because the peak of the effective area of the SGD is near $120 \mathrm{keV}$ [16], and the $122 \mathrm{keV}$ peak from the radiation source ${ }^{57} \mathrm{Co}$ was used for the energy calibration. We used the $194.5 \mathrm{keV}$ beam to examine the energy dependence of the polarimetric measurement capability of the Compton camera. For each energy, the Compton camera was rotated along the beam axis using $Y$-axis rotation stage to evaluate the camera response for various directions of the polarization vector. The experimentally set polarization angles $\left(\varphi_{\mathrm{pol}}\right)$ with respect to the camera's $X$-axis were $0^{\circ}, 22.5^{\circ}, 45^{\circ}, 90^{\circ}$, and $180^{\circ}$ (see Figures 2 and 4). We primarily measured the data for $\varphi_{\text {pol }}=0^{\circ}-90^{\circ}$ because the camera has a $90^{\circ}$-symmetric structure around the beam axis. To check the consistency of the experimental setup, we also obtained data for $\varphi_{\text {pol }}=180^{\circ}$, which should be consistent with that of $\varphi_{\text {pol }}=0^{\circ}$. For each angle, we aligned the camera with the beam so that the incident beam ran through the same spot of the camera and along the camera's $Z$-axis. The aligned spot was set at a pixel whose center was located $4.8 \mathrm{~mm}$ from the center of the Si layers along the camera's $X$ - and $Y$-axes (see also Figures 2 and 4 ) to avoid gaps between the bottom CdTe sensors under the Si layers because each CdTe layer is composed of $2 \times 2$ sensors (see Section 3.1).

The beam size was approximately $0.8 \mathrm{~mm}$ wide and $1.4 \mathrm{~mm}$ high. The intensity of the beam was kept constant for each energy. The event rate of the camera (either single hit event or multiple hits event) was $\sim 640 \mathrm{~Hz}$; the background rate (environmental radio activities) was $\sim 60 \mathrm{~Hz}$ so that the net event rate was $\sim 580 \mathrm{~Hz}$, giving the reconstructed event rate of $\sim 20$ and $\sim 30 \mathrm{~Hz}$ for 122.2 and $194.5 \mathrm{keV}$ beam, respectively (see also Section 5.1). Even though the precise incident beam intensity was not measured, we could roughly estimate the intensity to be approximately $580 \mathrm{~Hz} / s p /(1-d) \sim 1200$ photons/s, where $s p=0.92$ is the detector stopping power and $d=0.47$ is the dead-time ratio at $122.2 \mathrm{keV}$. The dead-time ratio was very high due to the high-intensity incident beam 4 The integration time for each polarization angle was approximately

\footnotetext{
4 Although the dead-time rate was high, we measured the live precisely (at the $\leq 2 \%$ level) using a "preudo trigger" function [16]. Therefore the impact on the validation of the
} 
80 min. The integration time was determined to obtain the modulation factor with a statistical error of $\leq 1 \%$. To check whether the experiment was reproducible, we measured the camera responses for $\varphi_{\text {pol }}=0^{\circ}$ at the beginning and end of the experiment. Both data sets were consistent within statistical errors at the energies of 122.2 and $194.5 \mathrm{keV}$.

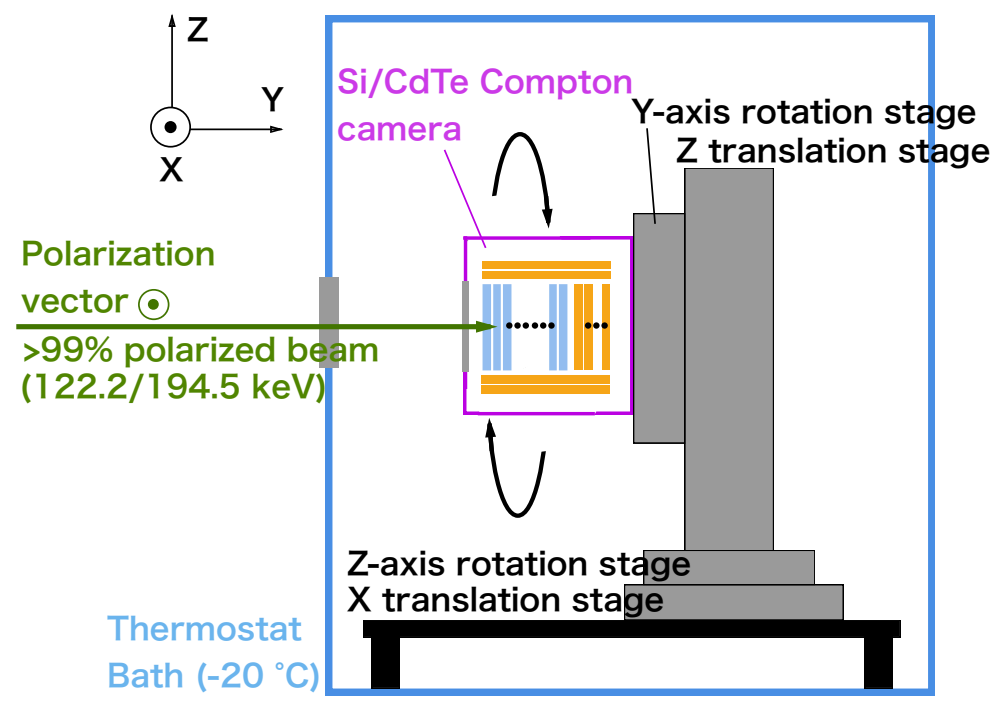

Figure 3: Schematic view of the experimental setup at Beamline BL08W.

Table 1: Stages used for the experiment

\begin{tabular}{|l|l|r|}
\hline Sort & Model number & Resolution \\
\hline X translation stage & XA16A-R1 & $1(\mu \mathrm{m} / \mathrm{step})$ \\
Z translation stage & ZA16A-X1 & $1(\mu \mathrm{m} / \mathrm{step})$ \\
Y-axis rotation stage & RA16A-WH & $2 \times 10^{-3}(\mathrm{deg} / \mathrm{step})$ \\
Z-axis rotation stage & RA10A-T01 & $5 \times 10^{-4}(\mathrm{deg} / \mathrm{step})$ \\
\hline
\end{tabular}

All stages were supplied by Kohzu Precision Co. Ltd. (Japan) modified for use at a temperature of $-20^{\circ} \mathrm{C}$.

instrument and simulator is small. 


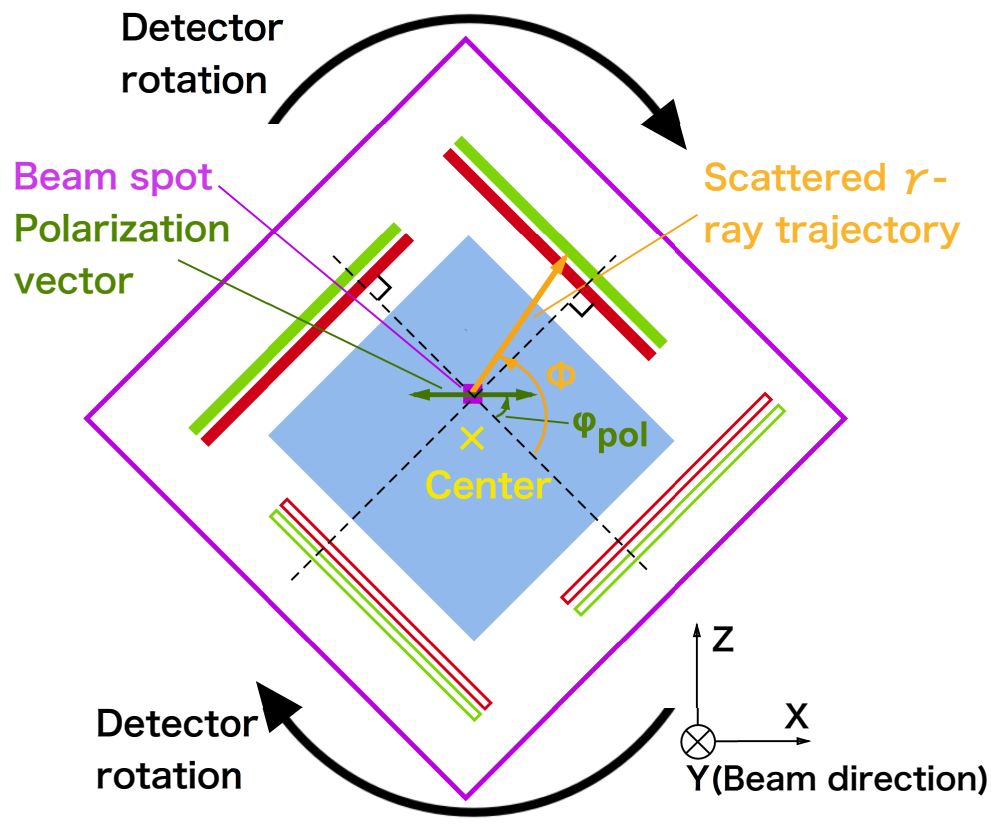

Figure 4: Top view of the Compton camera. The definitions of the azimuthal scattering angle $\phi$ and the polarization angle $\varphi_{\text {pol }}$, measured with respect to the camera's $X$-axis, are shown (see also Figure 2). The coordinate system of the experiment is the same as that in Figure 3 The inner and the outer four side CdTe layers are drawn as rectangles in red and green, respectively. The CdTe layers that are relatively closer to the incident beam spot are filled in with red or green, while the other relatively-distant layers are filled in with white (see Section 5.2). 


\section{Analysis and Results}

In this section, we demonstrate and evaluate the polarimetric performance of the SGD Compton camera. First, we produce the simulation data and the $N_{\text {cor }}(\phi)$ distribution and fit it with the model curve as described in Section2 to understand the polarimetric response of the SGD Compton camera. Then, we compare the experimental data with the simulation data to tune the MC simulation and to evaluate the systematic uncertainty of the SGD Compton camera. Finally, we evaluate the sensitivity of the polarized signal from a wellknown celestial object, the Crab Nebula.

\subsection{Event Selection}

To measure the beam polarization, we selected the reconstructed events (see also Section 3.2) that met the following conditions: (i) The deposited energies in the $\mathrm{Si}$ sensor were $5-40 \mathrm{keV}$ and $5-80 \mathrm{keV}$ for the 122.2 and $194.5 \mathrm{keV}$ beam, respectively. (ii) The measured total deposit energy was within $10 \mathrm{keV}$ of the incident beam energy (122.2 or $194.5 \mathrm{keV}$ ). (iii) The incident $\gamma$ ray was scattered by the Si sensors and then absorbed by the side CdTe sensors. (iv) The hit position in the Si sensor was at the pixel of the beam spot. Even though including the bottom CdTe sensors increases the number of events, we only used the side CdTe sensors as an absorber in this analysis for simplicity. We note that including the events with hits in bottom CdTe sensors is an important next step for fully validationg the instrument and simulator, and is also crucial to improve the statistics of the observational data. The selected events were approximately $6 \%$ and $4 \%$ of all the detected events from the beam (either single hit event or multiple hits event) at 122.2 and $194.5 \mathrm{keV}$, respectively. 


\subsection{Simulation Data}

We created simulation data in which the incident $\gamma$ rays went along the $Y$-axis with an energy of $122.2 \mathrm{keV}$, a physical size of $0.8 \times 1.4 \mathrm{~mm}^{2}$, and a polarization degree of $100 \%$ with $\varphi_{\text {pol }}=0^{\circ}$ with respect to the camera's $X$ axis, as shown in Figure 4. First, we set the incident beam spot to the center of the Si layers to understand the basic polarimetric response of the camera. The simulated data were selected under the conditions described in Section 5.1 .

For each selected event, we calculated the azimuthal scattering angle $(\phi)$ with respect to the camera's $X$-axis using the scattered $\gamma$-ray trajectory (see Figures 2 and 4 ). The bin size of $\phi$ (not uniform) was $\sim 6^{\circ}$, which corresponds to the pixel size of the CdTe sensor $(3.2 \mathrm{~mm})$. We calculated $\phi$ for the layers of the side CdTe detectors; each side CdTe detector has 16 pixels along the $\phi$ direction so that the total number of angle bins was 16 pixels $\times 8$ layers $=128$. In this analysis, we divided the data from the eight side CdTe layers into two groups: the data from inner four CdTe layers (hereafter the "inner layer data") and the data from the outer ones (hereafter the "outer layer data"), as shown in Figure 4 The top panel of Figure 5 shows the obtained azimuthal angle distribution. This distribution corresponds to $N_{\text {obs }}(\phi)$ in Section2. The distribution of the inner layer data is systematically larger than that of the outer layer data, because the scattered $\gamma$ rays first enter the inner CdTe layers and could be absorbed without reaching the outer $\mathrm{CdTe}$ layers. The gaps near $-120^{\circ},-30^{\circ}$, and $60^{\circ}$ correspond to the absence of the side CdTe detectors (see Section 3.1). In addition, we can see a dependency of the azimuthal scattering angle on the polarization vector, i.e., counts near $-90^{\circ}$ and $90^{\circ}$ are higher than those near $0^{\circ}$ and $180^{\circ}$.

To obtain a smooth modulation curve that follows the sinusoidal function of Equation (4), we need to correct the obtained $N_{\text {obs }}(\phi)$ by the detector response for nonpolarized beam $\left[N_{\text {iso }}(\phi)\right.$; see Equation (3)]. Therefore, we manufactured the same simulation data as above except with a nonpolarized incident beam. The azimuthal angle distribution of the simulated data is shown in the middle panel of Figure5. The distribution is bumpy, since $\sim 10 \%$ of the side CdTe pixels did not work in the experiment, primarily because of the bump-bonding disconnections between the sensors ( $\mathrm{Si}$ or $\mathrm{CdTe}$ ) and the readout board. We note that this is taken into account in the simulation, and the fraction of the bad pixels is much lower for the flight-model SGDs. Using these curves, we obtained the corrected distribution, $N_{\text {cor }}(\phi)$, as shown in the bottom panel of Figure5. The obtained $N_{\text {cor }}(\phi)$ was well fit using Equation (4), as shown in the bottom panel of Figure (5). The obtained parameters were $Q=0.66$ and $\phi_{0}=0.0^{\circ}$. We also conducted the same analyses for the data at $194.5 \mathrm{keV}$, and the obtained parameters were $Q=0.60$ and $\phi_{0}=0.1^{\circ}$. Therefore, we confirmed the analysis procedure.

Next, we generated the same simulation data as above at $122.2 \mathrm{keV}$, except that the incident beam spot was set to that of the experiment, which was off center by $4.8 \mathrm{~mm}$ along camera's $X$ - and $Y$-axis (see Figure2). Using the same analysis as above, we obtained $N_{\text {cor }}(\phi)$, as shown in Figure6 The fitting with Equation (4) resulted in $Q=0.66$ and $\phi_{0}=-0.1^{\circ}$. Even though the obtained 
values were nearly consistent with those for the beam irradiated at the center of the Si sensors, the top and bottom panels of Figure 6 show that the amplitudes of the curve near $-90^{\circ}$ and $0^{\circ}$ are larger than the other peaks $\left(90^{\circ}\right.$ and $180^{\circ}$, respectively). This is because the differential cross section [Equation (10)] depends not only on $\eta$ but also on $\theta$. Strictly speaking, Equation (4) can be applied to the distribution where the integration of $d \sigma / d \Omega$ in the $\theta$ space is independent of $\phi$. If this is not the case, $N_{\text {cor }}(\phi)$ deviates from the model curve. In this simulation, the incident beam spot is off center so that the distance to the side CdTe detectors from the spot systematically changes in the $\phi$ direction. This means that the viewing angle of $\theta$ changes in the $\phi$ direction. Such a $\phi$ dependency explains why the amplitude $(Q)$ of the $N_{\text {cor }}(\phi)$ distribution depends on $\phi$. To compensate for this $\phi$-dependece, we divided the distribution into two sets based on the distance to the side CdTe detectors: "data set $1^{\prime \prime}\left(-115^{\circ}<\phi<50^{\circ}\right)$ and "data set $2 "\left(\phi<-115^{\circ}\right.$ and $\left.\phi>50^{\circ}\right)$. The side CdTe detectors of the former set are more distant from the incident beam spot than those of the latter set. Note that we divided the inner and outer groups in the same way for simplicity. We then modified the model curve from Equation (4) to

$$
N_{\text {cor }}(\phi)=\left\{\begin{array}{lr}
A\left(1+Q_{1} \cos 2\left(\phi-\phi_{0}-\pi / 2\right)\right) & (\text { dataset } 1) \\
A\left(1+Q_{2} \cos 2\left(\phi-\phi_{0}-\pi / 2\right)\right) & (\text { dataset } 2)
\end{array}\right.
$$

Different parameters were used for the amplitude $\left(Q_{1,2}\right)$ to explain the $\phi$ dependency.

The best-fit curve is shown in the top panel of Figure 6 (the magenta line). The bottom panel demonstrates that the residuals decrease with respect to the original curve. There still remain small but statistically significant residuals (e.g., those at $\phi \sim 0^{\circ}$ ), likely because the modified model function is still not perfect. In this experiment, our goal was to evaluate the polarimetric performance of the Compton camera, in particular, the systematic uncertainty by comparing the experimental and simulation data, and Equation (9) is sufficient for such a purpose. The obtained parameters were $Q_{1}=0.68, Q_{2}=0.64$, and $\phi_{0}=0.0^{\circ}$ at $122 \mathrm{keV}$, while those for $193 \mathrm{keV}$ were $Q_{1}=0.64, Q_{2}=0.59$, and $\phi_{0}=-0.3^{\circ}$. Note that dividing the data is not necessary in orbit, because the $\gamma$ rays enter the entire area of the Si layers and the geometrical asymmetry is canceled. 


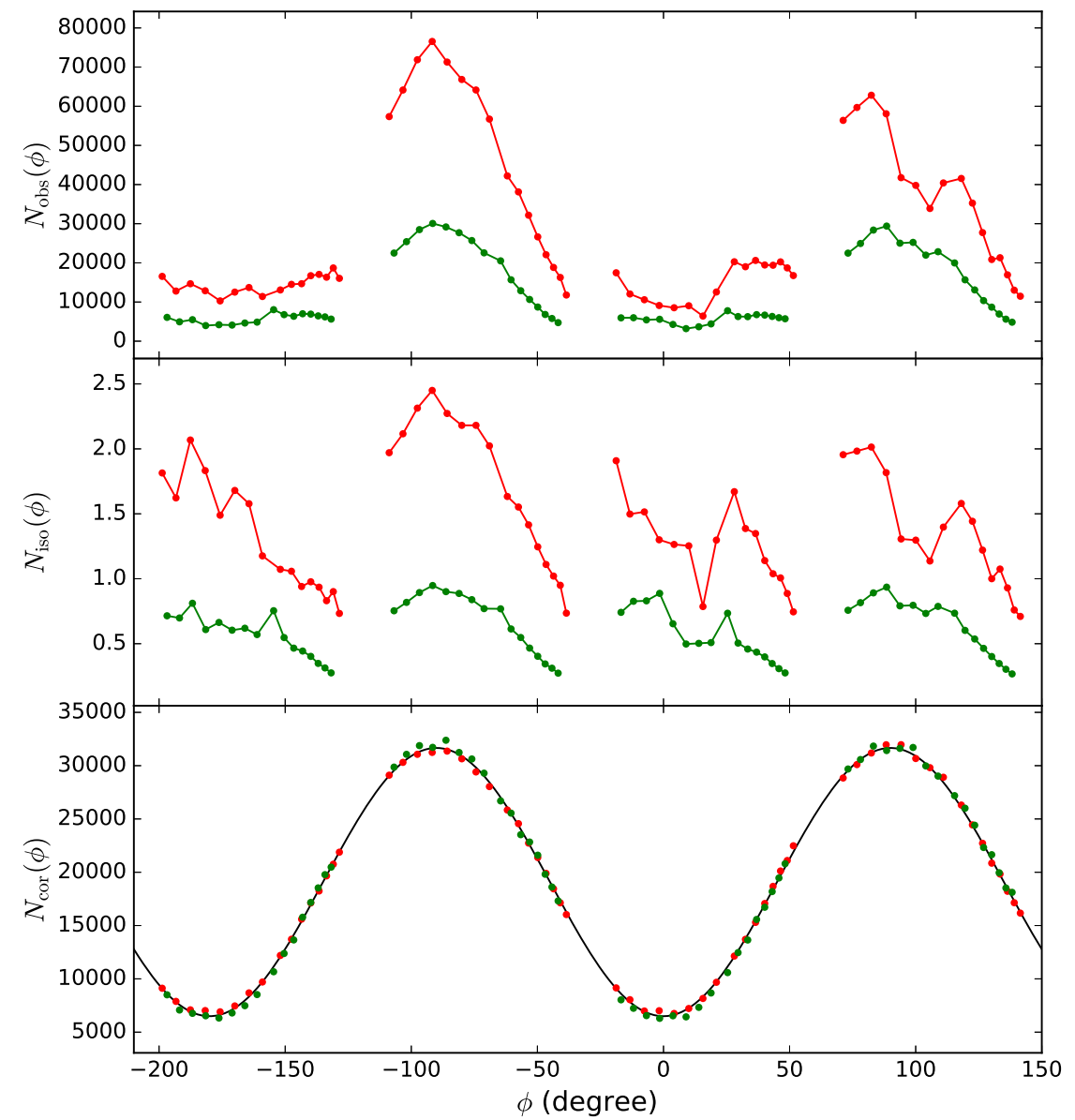

Figure 5: (Top) Azimuthal angle distribution of the simulated 100\%-polarized data at $\varphi_{\text {pol }}=0^{\circ}$ for a beam energy of $122.2 \mathrm{keV}$. The incident beam spot is at the center of the Si layers. The red and green points represent data from the inner and outer layers of the side CdTe detectors, respectively. The distribution corresponds to the $N_{\text {obs }}(\phi)$ distribution in Equation (3). Note that the statistical errors are smaller than the marker size. (Middle) Azimuthal angle distribution of the simulated nonpolarized beam corresponding to the $N_{\text {iso }}(\phi)$ distribution. (Bottom) The $N_{\text {cor }}(\phi)$ distribution obtained from $N_{\text {obs }}(\phi)$ and $N_{\text {iso }}(\phi)$ [see Equation (3)]. The black line represents the best-fit model curve of Equation (4). 


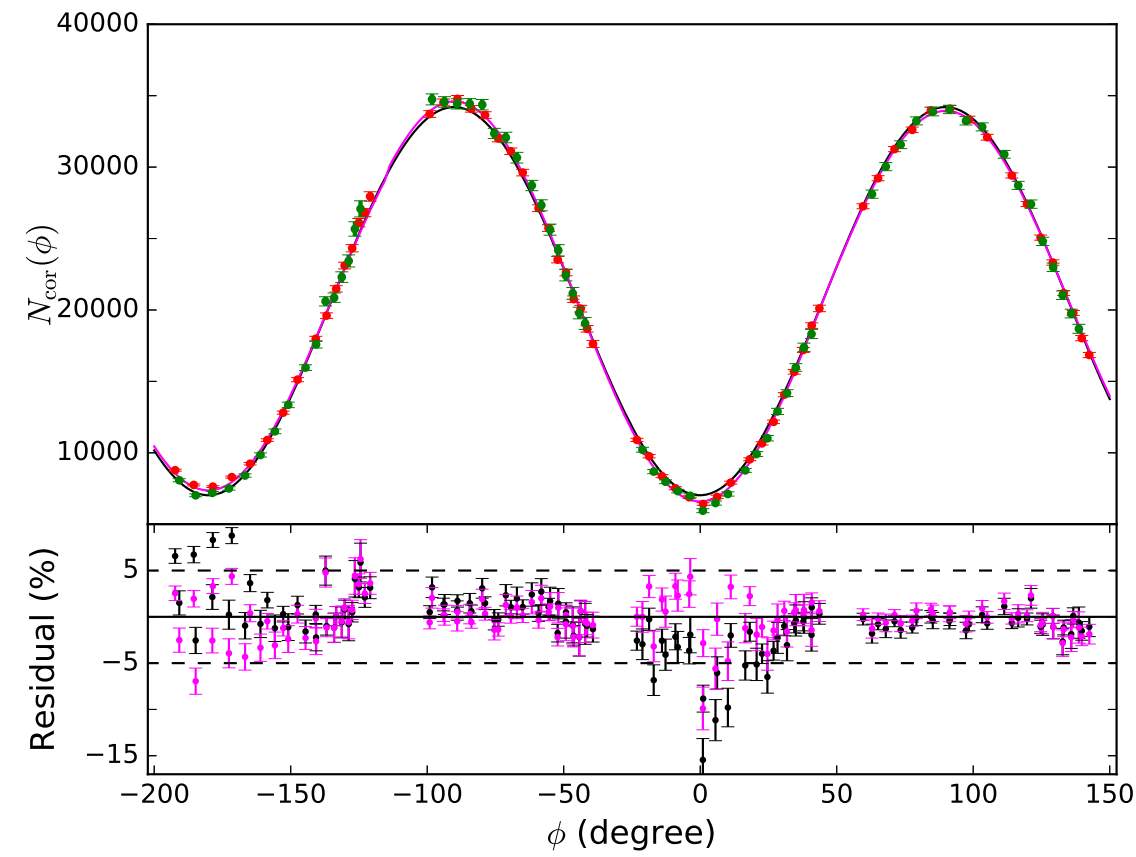

Figure 6: (Top) Same as the bottom panel of Figure5 except that the incident beam spot is set to that of the experiment. Note that most error bars are smaller than the marker size. The black and magenta lines are the best-fit curves of Equations (4) and (9) respectively. (Bottom) The distributions of (experimental data - model)/model. The black and magenta points represent the residuals when fitted by Equations (4) and (8), respectively. 


\subsection{Experimental Data}

We derived the $N_{\text {cor }}(\phi)$ distribution for the experimental data in a similar fashion to that described in Section 5.2 and found that the best-fit values of $\phi_{0}$ were systematically smaller by $\sim 1^{\circ}$ than the experimentally-set angles for all $\varphi_{\text {pol }}$ angles $\left(0^{\circ}, 22.5^{\circ}, 45^{\circ}, 90^{\circ}\right.$, and $\left.180^{\circ}\right)$. Given that the experiment setup has an uncertainty of $\sim 1^{\circ}$ with respect to the rotation around the beam axis, we concluded that the discrepancies were due to misalignment. The average of discrepancy was $1.4^{\circ}$, and we used this value as the offset angle around the beam axis. Therefore, the $\varphi_{\text {pol }}$ angles of the experiment were $-1.4^{\circ}, 21.1^{\circ}$, $43.6^{\circ}, 88.6^{\circ}$, and $178.6^{\circ}$.

We then selected the experimental data under the conditions described in Section 5.1 and derived the $N_{\text {obs }}(\phi)$ distribution as shown in Figure 7 for $\phi_{\mathrm{pol}}=1.4^{\circ}$. We also superimposed the distribution of the simulation data (for $\phi_{\text {pol }}=1.4^{\circ}$ ) with the $100 \%$ polarized beam in the figure. The parameters of the simulation data were the same as those in Section 5.2 except that the polar angle of the incident beam was set to $1^{\circ}$ with respect to the normal of the $\mathrm{Si}$ layers to reproduce the misalignment between the beam and the camera in the experiment. We also simulated data where the incident beam was exactly perpendicular to the camera; the difference between two data sets was up to $5 \%$ per bin in the azimuthal angle distributions, and the discrepancy of the best-fit parameters was less than $1 \%$ because the difference in $N_{\text {obs }}(\phi)$ was corrected by dividing by $N_{\text {iso }}(\phi)$. We unused angle bins of $-127^{\circ}--121^{\circ},-46^{\circ}--39^{\circ}$, $35^{\circ}-44^{\circ}$, and $133^{\circ}-143^{\circ}$, where the trajectories of the scattered photons go through the ASICs before reaching the side CdTe sensors. The mass model of the ASIC in the simulation has some uncertainty (see Section 3.3), and therefore, we decided not to simulate a precise response for these azimuthal angles but instead to simply remove the data from the analysis.

We also found that the total-count ratio of the outer layer data to the inner layer data in the simulation was systematically larger than that in the experiment. This is likely because the mass model of the materials between the inner and outer layer detectors has some uncertainties in the simulation so that the $\gamma$ rays between the detectors are less absorbed and/or scattered in the simulation than those in the real camera. We compared the data for all $\varphi_{\text {pol }}$ and found that the total-count ratio differs systematically (by $\sim 10 \%$ ). Improving the mass model is important to better reproduce the data with simulation, and one of next steps we will persue. In this analysis, however, we normalized the inner and outer layer data separately in the simulation to those in the experimental data and examined the remaining systematic uncertainty. Figure 7 shows the obtained experimental and simulated $N_{\text {obs }}(\phi)$ distributions at $\varphi_{\text {pol }}=-1.4^{\circ}$ and an energy of $122.2 \mathrm{keV}$. Even after normalization, the figure indicates that the residuals between the experimental and the simulation data are larger than those expected from the statistical errors. Such discrepancies are due to uncertainties in the detailed responses of each pixel in the simulation (e.g., the energy resolution and threshold) and should be considered as systematic uncertainties. To evaluate the systematic uncertainty quantitatively, we calculated the chi square value divided by the degree of freedom, $\chi^{2} /$ dof, between the experimental data 
and the simulation data for ten data sets (the inner and the outer data at the five $\varphi_{\text {pol }}$ angles). The ten calculated values range from $47.2 / 51$ to $122.4 / 51$, which contain statistically unacceptable values at the $99 \%$ confidence level. For data sets with large $\chi^{2} /$ dof, we added a uniform systematic error ratio to each $\phi$ angle bin to reduce $\chi^{2} /$ dof to $\simeq 1$. The added errors were at most $3 \%$ for ten data sets. Using the same procedure, the systematic errors at $194.5 \mathrm{keV}$ were evaluated to be $0 \%-3 \%$ for the ten data sets. Therefore, the systematic errors are similar for 122.2 and $194.5 \mathrm{keV}$. Note that the typical statistical errors were approximately $4 \%$ per $\phi$ angle bin. Therefore, the simulator reproduces the shape of the experimental distribution within a systematic uncertainty of $\leq 3 \%$ except for the unused angle ranges. We thus achieved systematic uncertainty as small as what obtained for the SGD prototype [18], using the Compton camera whose design is the same as that of the flight hardware. This uncertainty is much smaller than that of INTEGRAL/SPI in orbit evaluated in [27].

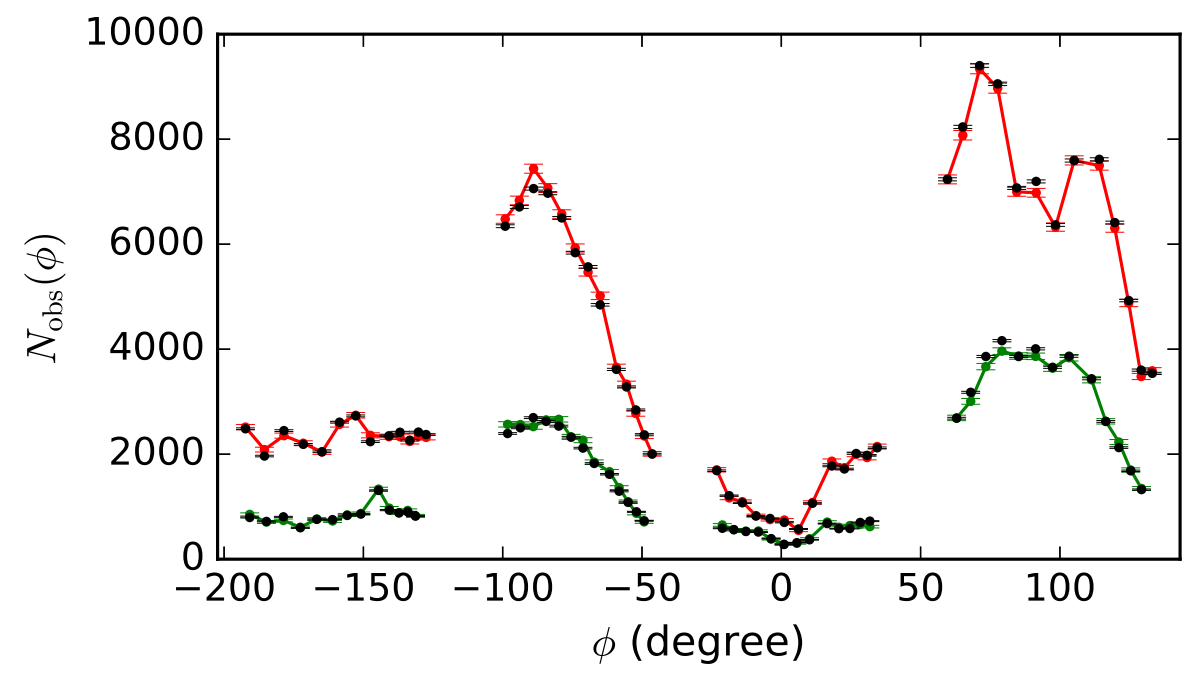

Figure 7: The $N_{\mathrm{obs}}(\phi)$ distribution at $\varphi_{\mathrm{pol}}=-1.4^{\circ}$ and an energy of $122.2 \mathrm{keV}$. The red and green points represent the inner and outer layer data of the experiment, respectively, while the superimposed black points show the simulated data. The simulated data are normalized to the total angular counts of the experimental data.

We obtained the $N_{\text {cor }}(\phi)$ distribution for the experimental data using the simulated $N_{\text {iso }}(\phi)$ distribution for all setups and fit them by Equation (9). We used the simulated $N_{\text {iso }}(\phi)$, because it is difficult to experimentally measure the response for nonpolarized $\gamma$-rays with the same condition. As described above, the total count ratio of $N_{\text {obs }}(\phi)$ for the outer layer data to the inner layer data in the simulation is larger than that in the experiment. This relationship is also true for $N_{\text {iso }}(\phi)$. If we use Equation (3) without corrections, the average value of $N_{\text {cor }}(\phi)$ for the outer layer data is smaller than that for the inner one. 
Therefore, we normalized the average of the outer layer data to that of the inner layer data. Figures 8 and 9 show the obtained modulation curves for 122.2 and $194.5 \mathrm{keV}$, respectively. The best-fit parameters are summarized in Table2 Even though the values of $\chi^{2} /$ dof are systematically larger than 1 , the model curves reproduce the observed data points well. This suggests that the bestfit parameters are good indicators of the polarimetric analyzing power of the Compton camera.

Table 2 demonstrates that the measured polarization angles $\left(\phi_{0}\right)$ are nearly consistent with the experimentally-set values. We calculated the differences

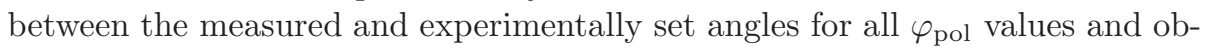
tained a standard deviation of $0.2^{\circ}$ and $0.3^{\circ}$ for the 122.2 and the $194.5 \mathrm{keV}$ data respectively. The uncertainties are slightly higher than the nominal statistical errors of $\leq 0.2^{\circ}$. The obtained values of $Q_{1}$ and $Q_{2}$ range from 0.649 to 0.701 and 0.637 to 0.653 for the inner layer data and the outer layer data, respectively, for $122.2 \mathrm{keV}$, and from 0.610 to 0.651 and 0.564 to 0.592 for the inner layer data and the outer layer data, respectively, for $194.5 \mathrm{keV}$. We calculated the standard deviation between the five $\varphi_{\text {pol }}$ data and took it to be the uncertainty of the $Q$ values. Then we obtained $Q_{1}=0.677 \pm 0.018$ and $Q_{2}=0.642 \pm 0.007$ at $122.2 \mathrm{keV}$. The values at $194.5 \mathrm{keV}$ were calculated to be $Q_{1}=0.632 \pm 0.014$ and $Q_{2}=0.575 \pm 0.013$. The relative standard deviations are $1.1 \%-2.7 \%$ and are larger than the nominal statistical errors of $\sim 0.5 \%$, suggesting that the simulated response $\left(N_{\text {iso }}\right)$ and/or the camera itself has a relative systematic error of $\leq 3 \%$. We also evaluated $Q_{1}$ and $Q_{2}$ for the simulation data and confirmed that the simulation and experimental results were consistent within $3 \%$. Because the incident beam was nearly $100 \%$ polarized, the fitted values of $Q_{1,2}$ can be considered to be experimentaly determined analyzing power $\left(Q_{100}\right)$ of the camera. Given Equation (5), an uncertainty in $Q_{100}$ indicates the potential polarimetric analyzing power of the instrument. The above results show that the experimentally-determined analyzing powers are consistent with the simulated ones within an accuracy of $3 \%$. 
Table 2: Best-fit parameters of the model curve for all the setups in the experiment.

\begin{tabular}{c|c||cccc}
\hline $\begin{array}{c}\text { Energy } \\
{[\mathrm{keV}]}\end{array}$ & $\begin{array}{c}\varphi_{\text {pol }} \\
{[\mathrm{deg}]}\end{array}$ & $Q_{1}$ & $Q_{2}$ & $\begin{array}{c}\phi_{0} \\
{[\mathrm{deg}]}\end{array}$ & $\chi^{2} / \mathrm{dof}$ \\
\hline 122.2 & -1.4 & $0.679 \pm 0.003$ & $0.637 \pm 0.003$ & $-1.26 \pm 0.13$ & $235.7 / 99$ \\
& 21.1 & $0.685 \pm 0.005$ & $0.639 \pm 0.004$ & $21.27 \pm 0.19$ & $161.4 / 99$ \\
& 43.6 & $0.701 \pm 0.007$ & $0.653 \pm 0.005$ & $43.30 \pm 0.16$ & $166.2 / 99$ \\
& 88.6 & $0.649 \pm 0.004$ & $0.648 \pm 0.004$ & $88.81 \pm 0.18$ & $132.4 / 99$ \\
& 178.6 & $0.675 \pm 0.005$ & $0.637 \pm 0.004$ & $178.51 \pm 0.19$ & $120.0 / 99$ \\
\hline 194.5 & -1.4 & $0.629 \pm 0.004$ & $0.565 \pm 0.004$ & $-1.58 \pm 0.18$ & $212.6 / 99$ \\
& 21.1 & $0.638 \pm 0.005$ & $0.568 \pm 0.005$ & $21.5 \pm 0.2$ & $140.2 / 99$ \\
& 43.6 & $0.651 \pm 0.008$ & $0.592 \pm 0.005$ & $43.76 \pm 0.18$ & $177.0 / 99$ \\
& 88.6 & $0.610 \pm 0.005$ & $0.590 \pm 0.004$ & $88.7 \pm 0.2$ & $147.5 / 99$ \\
& 178.6 & $0.633 \pm 0.005$ & $0.564 \pm 0.005$ & $178.7 \pm 0.2$ & $158.8 / 99$ \\
\hline
\end{tabular}

Only statistical errors are shown. 


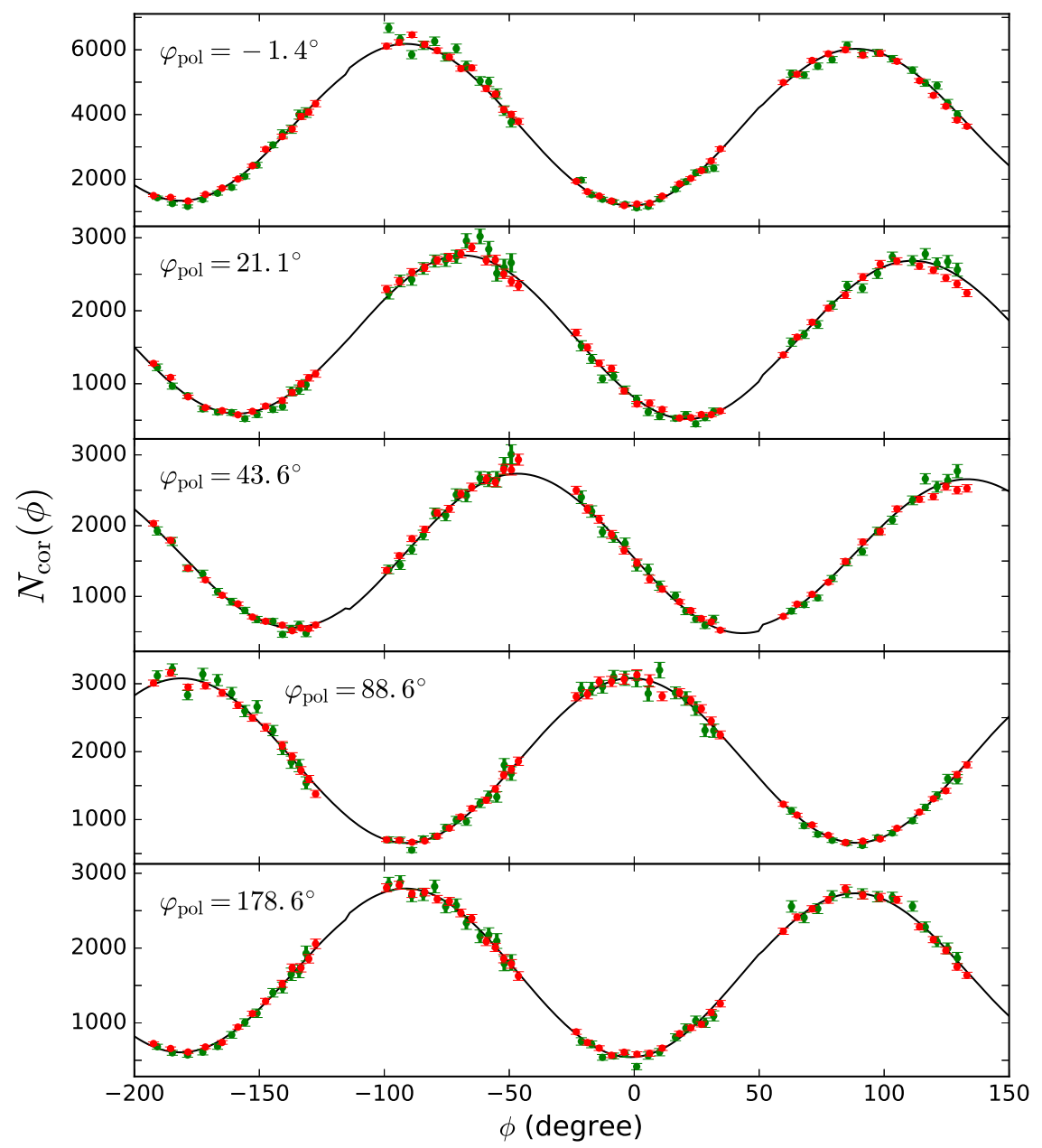

Figure 8: The corrected azimuth angle distributions for $\varphi_{\text {pol }}=-1.4^{\circ}, 21.1^{\circ}, 43.6^{\circ}$, $88.6^{\circ}$, and $178.6^{\circ}$ for a beam energy of $122.2 \mathrm{keV}$. The outer layer data are normalized to the average of the inner layer data. The best-fit curves modeled by Equation (9) are also plotted in black. Note that the model curves have discontinuous values at $\phi$ of $-115^{\circ}$ and $50^{\circ}$ (see text for details). 


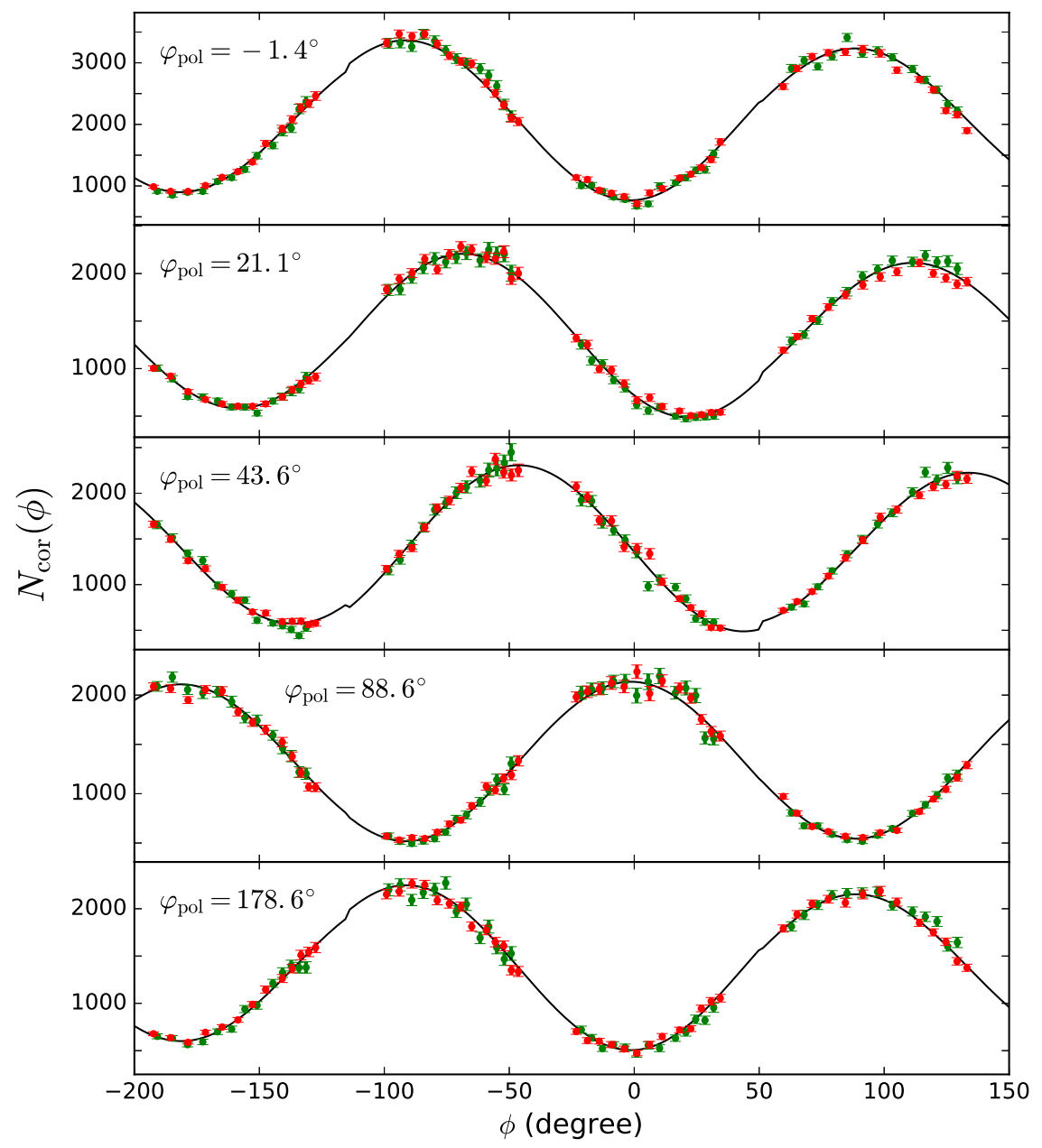

Figure 9: The same as Figure 8 but for a beam energy of $194.5 \mathrm{keV}$. 


\subsection{Polarization Sensitivity for a Celestial Object}

Finally we evaluated the polarimetric capability of the SGD on board the Hitomi satellite using an MC simulation. The minimum detectable polarization at the $99 \%$ confidence level can be calculated as

$$
\mathrm{MDP}_{99}=\frac{4.29}{Q_{100} \times R_{S}} \sqrt{\frac{R_{S}+R_{B}}{T}},
$$

where $R_{S}$ and $R_{B}$ are the source and the background count rates respectively, and $T$ is the observation time 28]. As a typical observation, we considered one of the brightest X-ray sources, the Crab Nebula, whose spectrum is assumed to be a power law, $F(E)=K \times E^{-\Gamma}$, with a normalization of $K=11.6$ photons $\mathrm{s}^{-2} \mathrm{~cm}^{-2}$ at $1 \mathrm{keV}$ and a photon index of $\Gamma=2.1$, where the energy $E$ is given in $\mathrm{keV}$. Note that the Carb Nebula is so bright that the background flux is almost negligible. In the simulation, we assumed that the incident $\gamma$ rays were perpendicular to the Si layers and uniformly entered the entire surface of the Si layer. The simulated $Q_{100}$ were calculated to be 0.70 and 0.61 in the energy bands of $60-100$ and $100-200 \mathrm{keV}$, respectively. The corresponding count rates of the SGD were expected to be 1.3 counts $^{-1}$ and 0.9 counts s$^{-1}$, respectively 5 With an observation time of $100 \mathrm{ks}$, the $\mathrm{MDP}_{99}$ were evaluated to be $1.7 \%$ and $2.3 \%$ in the energy bands of $60-100$ and $100-200 \mathrm{keV}$, respectively. This is much smaller than the reported polarization fraction of the Crab Nebula $(\sim 50 \%$ above $\sim 100 \mathrm{keV}[27,29])$. Since the relative uncertainty of $Q_{100}$ is only $\leq 3 \%$ as described in Section 5.3, it does not affect the MDP shown above. We note that in order to measure weak polarizations, possible fake modulations due to the systematic uncertainty could affect the sensitivity and should be investigated from ground and in-orbit data. For less bright objects, the uncertainty in the background should also be taken into account.

\footnotetext{
${ }^{5}$ The count rates are calculated by WebPIMMS: https://heasarc.gsfc.nasa.gov/cgibin/Tools/w3pimms/w3pimms.pl
} 


\section{Summary}

Gamma-ray polarization is a unique probe into the geometry of the $\gamma$-ray emission process in celestial objects. The Soft Gamma-ray Detector onboard the X-ray observatory Hitomi is a $\mathrm{Si} / \mathrm{CdTe}$ Compton camera and is expected to be an excellent polarimeter, as well as a highly sensitive spectrometer because of its good angular coverage and angular resolution for Compton scattering and its low background. To demonstrate the polarimetric capability and to verify and calibrate the Monte Carlo simulation program, we carried out a beam test of the final prototype for the SGD Compton camera at the large synchrotron facility SPring-8. We divided the absorber into two parts, the inner and outer layers of the side CdTe sensors, and obtained modulation factors of 0.649-0.701 (inner part) and $0.637-0.653$ (outer part) at $122.2 \mathrm{keV}$ and $0.610-0.651$ (inner part) and $0.564-0.592$ (outer part) at $194.5 \mathrm{keV}$, at varying polarization angles with respect to the detector. The test results suggest that the systematic uncertainty of the modulation factor is as small as $\leq 3 \%$. Using the "calibrated" simulator, we evaluated the polarimetric sensitivity of the SGD in orbit and obtained the minimum detectable polarization (with $99 \%$ confidence) of $1.7 \%$ and $2.3 \%$ in the energy bands of 60-100 and 100-200 keV, respectively, for the Crab Nebula with 100 ks observation in orbit.

\section{Acknowledgements}

This experiment was approved and conducted at the Spring- 8 for proposal No. 2015B1022. We appreciate the support of the SPring-8 staff. This work was partially supported by JSPS Grant-in-Aid for Scientific Research (A) Grant No. 24244014 and JSPS Grant-in-Aid for Scientific Research (B) Grant No. 25287059 . 


\section{References}

[1] P. Moran, G. Kyne, C. Gouiffès, P. Laurent, G. Hallinan, R. M. Redfern, A. Shearer, A recent change in the optical and $\gamma$-ray polarization of the Crab nebula and pulsar, MNRAS 456 (2016) 2974-2981.

[2] D. Yonetoku, T. Murakami, S. Gunji, T. Mihara, K. Toma, T. Sakashita, Y. Morihara, T. Takahashi, N. Toukairin, H. Fujimoto, Y. Kodama, S. Kubo, IKAROS Demonstration Team, Detection of Gamma-Ray Polarization in Prompt Emission of GRB 100826A, ApJL 743 (2011) L30.

[3] D. Yonetoku, T. Murakami, S. Gunji, T. Mihara, K. Toma, Y. Morihara, T. Takahashi, Y. Wakashima, H. Yonemochi, T. Sakashita, N. Toukairin, H. Fujimoto, Y. Kodama, Magnetic Structures in Gamma-Ray Burst Jets Probed by Gamma-Ray Polarization, ApJL 758 (2012) L1.

[4] D. Götz, S. Covino, A. Fernández-Soto, P. Laurent, Ž. Bošnjak, The polarized gamma-ray burst GRB 061122, MNRAS 431 (2013) 3550-3556.

[5] T. Takahashi, K. Mitsuda, R. L. Kelley, et al., The ASTRO-H x-ray observatory, Proc. SPIE 8443 (2012) 844312.

[6] T. Takahashi, K. Mitsuda, R. Kelley, et al., The ASTRO-H X-ray astronomy satellite, Proc. SPIE 9144 (2014) 914425.

[7] T. Takahashi, A. Awaki, T. Dotani, Y. Fukazawa, K. Hayashida, T. Kamae, J. Kataoka, N. Kawai, et al., Wide-band X-ray imager (WXI) and soft gamma-ray detector (SGD) for the NeXT mission, Proc. SPIE 5488 (2004) $549-560$.

[8] H. Tajima, R. Blandford, T. Enoto, et al., Soft gamma-ray detector for the ASTRO-H mission, Proc. SPIE 7732 (2010) 73216-773216-17.

[9] S. Watanabe, H. Tajima, Y. Fukazawa, et al., Soft gamma-ray detector for the ASTRO-H mission, Proc. SPIE 8443 (2012) 844326.

[10] Y. Fukazawa, H. Tajima, S. Watanabe, et al., Soft gamma-ray detector (SGD) onboard the ASTRO-H mission, Proc. SPIE 9144 (2014) 91442C.

[11] H. Tajima, T. Kamae, S. Uno, T. Nakamoto, Y. Fukazawa, T. Mitani, T. Takahashi, K. Nakazawa, Y. Okada, M. Nomachi, Low noise doublesided silicon strip detector for multiple-Compton gamma-ray telescope, in: J. E. Truemper, H. D. Tananbaum (Eds.), X-ray and Gamma-ray Telescopes and Instruments for Astronomy, SPIE 4851 (2002) 875-884.

[12] Y. Fukazawa, T. Nakamoto, N. Sawamoto, S. Uno, T. Ohsugi, H. Tajima, T. Takahashi, T. Mitani, T. Tanaka, K. Nakazawa, Development of lownoise double-sided silicon strip detector for cosmic soft gamma-ray Compton camera, Nucl. Instr. and Meth. A 541 (2005) 342-349. 
[13] S. Takeda, S. Watanabe, T. Tanaka, K. Nakazawa, T. Takahashi, Y. Fukazawa, H. Yasuda, H. Tajima, Y. Kuroda, M. Onishi, K. Genba, Development of double-sided silicon strip detectors (DSSD) for a Compton telescope, Nucl. Instrum. Methods A 579 (2007) 859-865.

[14] T. Takahashi, S. Watanabe, Recent progress in CdTe and CdZnTe detectors, IEEE Trans. Nucl. Sci. 48 (2001) 950-959.

[15] S. Takeda, H. Odaka, S. Ishikawa, S. Watanabe, H. Aono, T. Takahashi, Y. Kanayama, M. Hiromura, S. Enomoto, Demonstration of in-vivo multiprobe tracker based on a $\mathrm{Si} / \mathrm{CdTe}$ semiconductor Compton camera, IEEE Trans. Nucl. Sci. 59 (2012) 70-76.

[16] S. Watanabe, H. Tajima, Y. Fukazawa, et al., The Si/CdTe semiconductor Compton camera of the ASTRO-H Soft Gamma-ray Detector (SGD), Nucl. Instrum. Methods A 765 (2014) 192-201.

[17] H. Tajima, Gamma-ray polarimetry, Nucl. Instrum. Methods A 511 (2003) 287-290.

[18] S. Takeda, H. Odaka, J. Katsuta, S. Ishikawa, S. Sugimoto, Y. Koseki, S. Watanabe, G. Sato, M. Kokubun, T. Takahashi, K. Nakazawa, Y. Fukazawa, H. Tajima, H. Toyokawa, Polarimetric performance of $\mathrm{Si} / \mathrm{CdTe}$ semiconductor Compton camera, Nucl. Instrum. Methods A 622 (2010) 619-627.

[19] F. Lei, A. J. Dean, H. G. L, Compton polarimetry in gamma-ray astronomy, Space Science Review 82 (1997) 309.

[20] Y. Ichinohe, Y. Uchida, S. Watanabe, I. Edahiro, K. Hayashi, T. Kawano, M. Ohno, M. Ohta, S. U. Takeda, Y. Fukazawa, M. Katsuragawa, K. Nakazawa, H. Odaka, H. Tajima, H. Takahashi, T. Takahashi, T. Yuasa, The first demonstration of the concept of "narrow-FOV Si/CdTe semiconductor Compton camera", Nucl. Instrum. Methods A 806 (2016) 5-13.

[21] H. Odaka, S. Sugimoto, S. Ishikawa, et al., Development of an integrated response generator for $\mathrm{Si} / \mathrm{CdTe}$ semiconductor Compton cameras, Nucl. Instrum. Methods A 624 (2010) 303.

[22] S. Agostinelli, et al., GEAnt4 - a simulation toolkit, Nucl. Instrum. Methods A 506 (2003) 250.

[23] J. Allison, et al., Geant4 developments and applications, IEEE Transactions on Nuclear Science 53 (2006) 270.

[24] X. Maréchal, T. Hara, T. Tanabe, et al., Development of an elliptical multipole wiggler at SPring-8, Journal of Synchrotron Radiation 5 (1998) 431-433. 
[25] H. Yamaoka, N. Hiraoka, M. Itou, et al., Performance of bent-crystal monochromators for high-energy synchrotron radiation, Journal of Synchrotron Radiation 7 (2000) 69-77.

[26] M. Itou, M. Brancewicz, Y. Sakurai, Present Status of BL08W (2014), SPring-8 / SACLA Research Report 3 (2014) 124-129.

[27] A. J. Dean, D. J. Clark, J. B. Stephen, V. A. McBride, L. Bassani, A. Bazzano, A. J. Bird, A. B. Hill, S. E. Shaw, P. Ubertini, Polarized Gamma-Ray Emission from the Crab, Science 321 (2008) 1183.

[28] M. C. Weisskopf, R. F. Elsner, V. M. Kaspi, S. L. O’Dell, G. G. Pavlov, B. D. Ramsey, X-Ray Polarimetry and Its Potential Use for Understanding Neutron Stars, volume 357 of Astrophysics and Space Science Library, 2009.

[29] M. Forot, P. Laurent, I. A. Grenier, C. Gouiffès, F. Lebrun, Polarization of the Crab Pulsar and Nebula as Observed by the INTEGRAL/IBIS Telescope, ApJL 688 (2008) L29. 